\title{
Redes de Comunicação Sem Fio Cooperativas: Protocolos, Topologias e Desempenho
}

\section{Wireless Cooperative Networks: Protocols, Topologies and Performance}

\author{
Álvaro Ricieri Castro e Souza ${ }^{1}$; Mario Lemes Proença Jr²; Taufik Abrão ${ }^{3}$
}

\section{Resumo}

Este trabalho analisa os principais protocolos em redes sem fio cooperativas, buscando verificar potencialidades e desempenho das mesmas em relação à metodologia não cooperativa, além de técnicas que possam maximizar o desempenho, como por exemplo regras de combinação de sinal e seleção de relays. São feitas considerações acerca da complexidade computacional envolvida tanto nas topologias quanto nas técnicas de combinação e protocolos empregados nas redes cooperativas.

Palavras-chave: Redes sem fio cooperativas. Protocolos. Complexidade computacional.

\begin{abstract}
This work analyzes the most common wireless cooperative network protocols, verifying potentialities and performance relative to the non cooperative methodology, also techniques that can maximize the performance, such as combining signal rules and relay selection. Furthermore, considerations are provided for the computational complexity of topologies, combining techniques and protocols of cooperative networks.
\end{abstract}

Key words: Wireless cooperative networks. Protocols. Computational complexity.

\section{Introdução}

Com o constante crescimento da utilização de redes de comunicação, novos paradigmas e desafios são lançados aos pesquisadores, principalmente a necessidade de aumento da taxa de transmissão com restrição na largura de banda disponível. Para as redes sem fio e dispositivos móveis surgem novas problemáticas, como ampliação de área de cobertura, throughput (vazão de dados), problemas de canal (desvanecimento do sinal, interferências e obstáculos) e economia de recursos - sejam de hardware ou mesmo energia.

Tendo em foco este cenário surgem as pesquisas em redes cooperativas, que visam a utilização dos dispositivos ativos na rede como relays (retransmissores), obtendo desta forma diversidade semelhante à encontrada em dispositivos MIMO (Multiple Input Multiple Output) que, combinada a métodos de aproveitamento da mesma, é capaz de propiciar transmissões mais efetivas, com redução dos efeitos de canal (KRAMER; MARIĆ; YATES, 2006; NOSRATINIA; HUNTER; HEDAYAT, 2004) e melhoria em termos de potência (RODOPLU; MENG 1999; ZHOU et al., 2008), taxa de erro de bit (BER) e throughput.

Para que ocorra a cooperação entre os dispositivos é necessário o desenvolvimento e otimização de

\footnotetext{
1 Mestrando do Programa de Pós-Graduação em Computação, Universidade Estadual de Londrina; alvarorcsouza@gmail.com

2 Professor Adjunto do Departamento de Computação; proenca@uel.br

3 Professor Associado do Departamento de Engenharia Elétrica da Universidade Estadual de Londrina - DEEL-UEL; taufik@uel.br
} 
protocolos cooperativos. Dentre os mais conhecidos estão o Amplifica e Transmite (AF - amplify-andforward) (VIEN; NGUYEN 2010; YAN; YUE; HE, 2010; TORABI; HACCOUN; AJIB, 2010), no qual o sinal recebido pelo relay é amplificado e retransmitido ao destinatário, Decodifica e Transmite (DF - decode-and-forward) (SELVARAJ; MALLIK, 2010), onde o sinal recebido pelo relay é detectado e então transmitido ao destinatário, e Decodifica e Transmite com Codificação (DFC decode-and-forward with coding) (ZHANG et al., 2009), em que o sinal é detectado e decodificado pelo relay e, em caso de não-detecção de erro, é novamente codificado e transmitido ao destinatário.

Já com relação aos métodos de combinação de sinal, os principais métodos utilizados são Combinação por Seleção (SC - Selection Combining) (QUOC et al., 2008), no qual o sinal com maior SNR instantânea é escolhido para detecção, Combinação de Ganho Igualitário (EGC Equal Gain Combining) (IKKI; AHMED, 2009), na qual o sinal a ser detectado é composto igualmente por todos os sinais recebidos pelo destinatário, e Combinação de Máxima Razão (MRC - Maximalratio combining) (VIEN; NGUYEN, 2010), onde os sinais são ponderados de acordo a SNR instantânea dos mesmos, sendo o método mais eficiente de aproveitamento de diversidade, porém com a maior complexidade computacional.

Deste modo, este trabalho tem por objetivo o estudo do modelo cooperativo, tanto em termos de topologias e protocolos quanto dos métodos de combinação de sinais, além de demonstração de suas potencialidades por meio de resultados numéricos de simulação relativos às duas principais figuras de mérito empregadas na caracterização de sistemas de comunicação digital: taxa de erro de bit e probabilidade de queda da comunicação (outage). A análise se dará por meio de simulações computacionais dos protocolos e técnicas estudadas, visando demonstrar as potencialidades desta abordagem em relação ao modelo não cooperativo.
O trabalho está distribuído como segue. Na Seção 2 o modelo não-cooperativo e as condições de canal às quais as transmissões estão sujeitas são descritos; a Seção 3 trata do modelo cooperativo e dos principais protocolos de cooperação difundidos na literatura. A Seção 4 traz uma análise dos principais métodos de combinação de sinais, necessários ao adequado aproveitamento da diversidade gerada pelo modelo cooperativo, enquanto a Seção 5 descreve o método de simulação e da análise dos resultados obtidos. Finalmente, a Seção 6 traz as conclusões obtidas neste trabalho.

\section{Modelo não cooperativo}

O modelo não cooperativo é caracterizado por uma abordagem na qual cada dispositivo trata apenas de seus sinais, descartando transmissões de outros dispositivos não endereçadas para si mesmo. Tal abordagem é vista como um modo de se manter privacidade e segurança dos dados que trafegam na rede, requisitos básicos em qualquer modelagem.

O principal problema desta abordagem se dá no enfrentamento das condições de canal, tais como ruído e o desvanecimento (fading), sendo estas especialmente críticas em comunicações sem fio (STALLINGS, 2005). Um exemplo de método de enfrentamento das condições de canal são os dispositivos MIMO, que com a utilização de múltiplas antenas consegue propiciar diversidade às transmissões. Seu principal problema se dá na necessidade da utilização de mais antenas, o que pode ser inviável em determinados dispositivos.

O conceito de comunicação cooperativa introduz diversidade extra no combate às condições degradantes que o canal de rádio móvel estabelece. Neste paradigma, os dispositivos atuam também no modo retransmissor (além dos modos transmissor/ receptor), estabelecendo-se desta forma diversidade similar à existente no modelo MIMO sem a necessidade de inserção de mais antenas (KORAKIS et al., 2009), possibilitando o enfrentamento destas condições degradantes, principalmente no que se 
refere ao desvanecimento profundo. $\mathrm{Na}$ próxima seção serão discutidas questões práticas do modelo cooperativo, tais como protocolos cooperativos, capacidade e a problemática cooperativa, considerando o meio de transmissão sem fio sujeito a canais com desvanecimento Rayleigh.

\section{Modelo cooperativo e protocolos de cooperação}

A descrição do modelo cooperativo se dará a partir da figura 1, que descreve o modelo básico de uma rede cooperativa, com um dispositivo fonte (source), um relay e um receptor (destination).

Figura 1. Modelo básico de uma rede cooperativa.

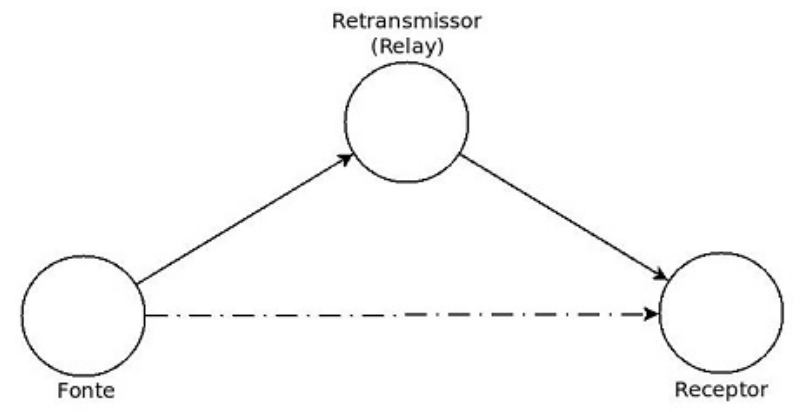

A abordagem cooperativa não determina qual método de acesso ao meio será utilizado, podendo ser adaptada para os modelos mais conhecidos CDMA (JAHROMI et al., 2009), TDMA (QUOC et al., 2008) e FDMA (SIRIWONGPAIRAT; SADEK; LIU, 2008). Como não esta no escopo deste trabalho a avaliação das técnicas de acesso ao meio, será considerado um modelo baseado em TDMA, tendo 2 time-slots. A escolha pelo método TDMA de acesso ao meio é dada por questões de simplicidade, dado que sua simulação e método de operação são mais triviais.

O nó fonte gera um sinal ss, segundo a seguinte equação:

$$
s_{s}=\sqrt{P_{s}} \cdot b_{i}
$$

com $P_{s}$ sendo a potência de transmissão da fonte e $b_{i}$ a informação transmitida, considerando a utilização de modulação BPSK (Binary Phase Shift Keying). A modulação BPSK foi escolhida por simplicidade. No entanto, a análise desenvolvida neste trabalho é facil e imediatamente estendida considerando-se outros formatos de modulação de elevada ordem, como por exemplo $M$-QAM, com $M \geq 4$.

O sinal ss é transmitido no primeiro time-slot e recebido tanto pelo receptor quanto pelo relay, conforme as equações:

$$
\begin{aligned}
& r_{s d}=\sqrt{P_{s}} \cdot b_{i} \cdot h_{s d}+\eta_{d} \\
& r_{s r}=\sqrt{P_{s}} \cdot b_{i} \cdot h_{s r}+\eta_{r}
\end{aligned}
$$

onde $\mathrm{h}_{\mathrm{sj}}$ refere-se ao ruído multiplicativo de canal entre o dispositivo fonte e o $j$-ésimo dispositivo (podendo $\operatorname{ser} j=d$, destino ou $j=r$, relay) e $\eta_{j}$ é o ruído de fundo no j-ésimo receptor (podendo ser $j=d$, destino ou $j=r$, relay).

O relay processa o sinal de acordo com o protocolo utilizado e, no segundo time-slot transmite o sinal ao destinatário, que combina os sinais de acordo com a técnica definida e então detecta a informação. Para a descrição dos protocolos estudados, será considerado um sistema com 1 fonte, $N$ relays $(N>$ $0)$ e 1 destinatário, além de ser baseado em TDMA com $N+1$ time-slots.

\section{Protocolo Amplifica e Transmite}

O primeiro protocolo cooperativo abordado é o amplifica e transmite. $\mathrm{O}$ funcionamento deste pode ser visto na figura 2 .

Figura 2. Representação do protocolo amplifica e transmite.

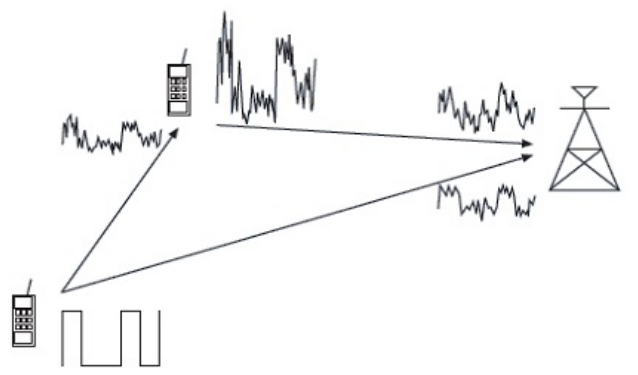


O primeiro time-slot ocorre de maneira similar à descrita anteriormente, com a transmissão e recepção do sinal s pelos relays e receptor. Como o próprio nome sugere, os relays quando baseados neste protocolo aplicam um fator de amplificação $G_{i}$ ao sinal $\mathrm{r}_{\text {sri }}$, obtendo o sinal a ser transmitido ao dispositivo receptor conforme a seguinte equação:

$$
s_{r_{i}}=G_{i} \cdot\left(\sqrt{P_{s}} \cdot b_{i} \cdot h_{s r_{i}}+\eta_{r_{i}}\right)
$$

A forma de cálculo do fator de ganho $G_{i}$ pode ser feito de duas maneiras distintas: com valor fixo, em casos nos quais não há informação de estado de canal (CSI - Channel-state-information), utilizandose normalmente o valor médio da atenuação no caminho fonte-i-ésimo relay (XU; ZHANG; ZHANG, 2010; HASNA; ALOUINI, 2004), ou com ganho variável, baseado no CSI, como em (HASNA; ALOUINI, 2002) e (LANEMAN; WORNELL, 2000). O fator de ganho neste trabalho baseia-se no desenvolvido em (YAN; YUE; HE, 2010), porém modificando-se a regra que determina a potência de transmissão do i-ésimo relay, $P_{r i}$. $\mathrm{O}$ cálculo do ganho $G_{i}$ é basicamente estabelecido pela raiz quadrada da razão entre a potência do relay pela potência de transmissão no nó origem:

$$
G_{i}=\underbrace{\frac{h_{s r_{i}}^{*}}{\left|h_{s r_{i}}\right|}}_{(\mathrm{I})} \cdot \underbrace{\sqrt{\frac{P_{r_{i}}}{P_{s} \cdot\left|h_{s r_{i}}\right|^{2}+\sigma_{r_{i}}^{2}}}}
$$

(I)

onde o termo (I) é essencialmente a correção dos erros de fase advindos do caminho fonte-relay, (.)* representa o operador complexo conjugado e o termo (II) se refere ao termo de amplificação do sinal, tal que $\mathbf{E}\left[\left|G_{i} \cdot r_{s r i}\right|\right]=P_{r i}$, sendo $\mathbf{E}[$.$] o operador$ esperança estatística.

Cada relay, então, transmite seu sinal amplificado para o destinatário, sendo o sinal recebido descrito como:

$$
r_{r_{i} d}=G_{i} \cdot\left(\sqrt{P_{s}} \cdot b_{i} \cdot h_{s r_{i}}+\eta_{r_{i}}\right) \cdot h_{r_{i} d}+\eta_{d}
$$

Visando aumentar a resistência deste protocolo às condições mais severas de canal, alguns trabalhos como (YAN; YUE; HE, 2010) sugerem a utilização de um threshold de $\operatorname{SNR}\left(\gamma_{r i}^{*}\right)$ para a retransmissão do sinal e/ou também para a recepção do $\operatorname{sinal}\left(\gamma_{\mathrm{d}}^{*}\right)$. Assim, caso a relação sinalruído recebido pelo i-ésimo relay não atingir um valor mínimo de SNR pré-estabelecido, não ocorre a retransmissão; e no caso da SNR instantânea do sinal retransmitido não alcançar o mínimo, é ignorado no processo de detecção. Esta estratégia visa a redução da probabilidade de transmissão de informações incorretas a partir da exclusão de sinais cujas SNRs sejam reduzidas, resultando assim em menores probabilidades de erro no receptor. A regra de cálculo da SNR instantânea é dada pela eq. (7), e a regra de decisão do sinal transmitido pela eq. (8).

$$
\begin{aligned}
& \gamma_{s r_{i}}=\Gamma_{s r_{i}} \cdot\left|h_{s r_{i}}\right|^{2} \\
& s_{r_{i}}= \begin{cases}G_{i} \cdot s_{r_{i}}, & \text { se } \gamma_{s r_{i}} \geq \gamma_{r_{i}}{ }^{*} \\
0, & \text { caso contrário }\end{cases}
\end{aligned}
$$

sendo $\Gamma_{s r_{i}}=\frac{P_{s}}{P_{\text {ruidor }_{i}}}$ a SNR média no $i$-ésimo relay, devido ao sinal transmitido no nó fonte (s).

As principais vantagens atribuídas a este protocolo são ordem de diversidade mais elevada (LANEMAN; WORNELL; TSE, 2001) e também complexidade computacional reduzida em relação a outros protocolos, devido à não-detecção do sinal.

\section{Protocolo Decodifica e Transmite}

Como o próprio nome sugere, este protocolo se diferencia do anterior no tratamento do sinal recebido em cada relay. Seu funcionamento pode ser deduzido através da figura 3. 
Figura 3. Representação do protocolo decodifica e transmite.

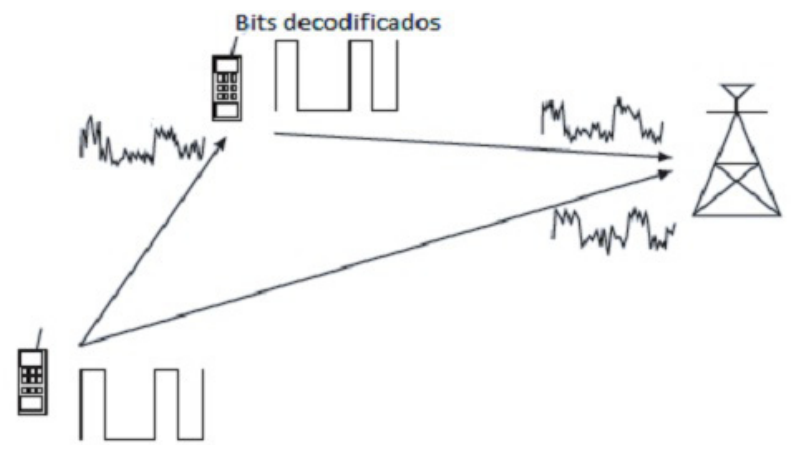

Como acontece no protocolo anterior, o nó fonte transmite o sinal para os nós destinatário e relays, com os sinais recebidos sendo descritos conforme as eqs. (2) e (3). Após a recepção do sinal, cada relay procede à detecção da informação transmitida. Considerando modulação BPSK, este processo pode ser descrito pela seguinte equação:

$$
\widehat{b_{i}}=\operatorname{sign}\left[\Re\left(h_{s r_{i}}^{*} \cdot r_{s r_{i}}\right)\right]
$$

Finalmente, cada relay transmite a informação detectada para o destinatário, segundo a seguinte equação:

$$
r_{r_{i} d}=\sqrt{P_{r_{i}}} \cdot \widehat{b_{i}} \cdot h_{r_{i} d}+\eta_{d}
$$

A principal vantagem desta abordagem sobre o anterior se dá na não-propagação dos efeitos de canal no sinal retransmitido (NOSRATINIA; HUNTER; HEDAYAT, 2004), porém pode ocorrer a detecção incorreta da informação transmitida, o que pode resultar em erro na detecção do sinal no receptor. Outras desvantagens estão na menor ordem de diversidade atingida e também na complexidade de detecção (dependente dos sistemas de modulação e acesso utilizados).

Assim como ocorre com protocolo anterior, técnicas são pesquisadas para reduzir a probabilidade de propagação/ocorrência de erros. A primeira destas abordagens é semelhante à descrita para o amplifica e transmite, com a utilização de thresholds de SNR (LEE; KIM, 2007; QUOC et al., 2008). A regra de decisão do sinal transmitido é dada pela eq. (11).

$$
s_{r_{i}}= \begin{cases}\sqrt{P_{r_{i}}} \cdot \widehat{b_{i}}, & \text { se } \gamma_{s r_{i}} \geq \gamma_{r_{i}}{ }^{*} \\ 0, & \text { caso contrário }\end{cases}
$$

Outra abordagem desenvolvida é a utilização de esquemas de codificação (DFC) (ALAZEM; FRIGON; HACCOUN, 2008; ZHANG; MA; TAFAZOLLI, 2008; NOSRATINIA; HUNTER; HEDAYAT, 2004; HUNTER; NOSRATINIA, 2006), com os quais a informação detectada pode ser checada quanto à existência de erros. Um exemplo desta abordagem pode ser vista em (NOSRATINIA; HUNTER; HEDAYAT, 2004), em que se utiliza codificação por meio da utilização de CRC (cyclic redundancy check). Desta maneira, cada relay é capaz de verificar se a informação recebida é coerente: dependendo dos bits trocados, a verificação do CRC pode ser dada como correta, porém os dados não estarem em conformidade com os originais. A vantagem do método reside na capacidade de identificar grande parte dos erros de detecção, porém incorre em maior complexidade em relação ao método anterior.

\section{Métodos de Combinação de Sinal}

Basicamente, há quatro métodos de combinação de sinais que podem ser empregados em qualquer tipo de diversidade (espacial, frequência, temporal, percurso, macro-diversidade, entre outras): combinação por seleção (SC), combinação por ganho igualitário (EGC), combinação de máxima razão (MRC) e combinação por regra empírica (ER). Neste trabalho serão explorados os métodos de combinação SC, EGC e MRC.

\section{Combinação por Seleção}

O método de combinação por seleção (SC Selection Combining) é caracterizado por escolher um dos sinais recebidos para o processo de detecção, utilizando como métrica a SNR instantânea $(\gamma)$ de cada sinal. Após receber os sinais tanto do nó 
fonte quanto dos relays, o nó destino calcula a SNR instantânea de cada transmissão e escolhe o sinal que atingir o maior valor para $\gamma$, de acordo com a regra definida em (12):

$$
r_{d}= \begin{cases}r_{s d}, & \text { se } \gamma_{s d}>\gamma_{r_{j} d}, j=1,2 \ldots N \\ r_{r_{i} d}, & \text { se } \gamma_{r_{i} d}>\gamma_{s d} \text { e } \gamma_{r_{i} d}>\gamma_{r_{j} d} \\ & i, j=1,2 \ldots N, i \neq j\end{cases}
$$

Como somente um sinal é escolhido para detecção, a SNR instantânea para o método SC é definida como a SNR do sinal detectado, ou seja:

$$
\gamma_{S C}=\gamma_{r_{d}}
$$

A principal vantagem da combinação por seleção se dá na baixa complexidade computacional exigida em relação à outros métodos (PROAKIS, 2001), pois apenas um sinal precisa ter todos os seus coeficientes estimados. Entre as desvantagens pode se citar o aproveitamento marginal da diversidade propiciada.

\section{Combinação de Ganho Igualitário}

$\mathrm{O}$ método de combinação por ganho igualitário (EGC - Equal Gain Combining) é um método que, como o próprio nome sugere, combina os diversos sinais disponíveis no destinatário utilizando como métrica pesos idênticos, realizando detecção coerente de cada um dos sinais (ou seja, remoção de fase).

O sinal a ser detectado pode ser definido como:

$$
\begin{aligned}
& r_{d}=w_{1} \cdot \frac{h_{s d}^{*}}{\left|h_{s d}\right|} \cdot r_{s d}+\sum_{i=1}^{N} w_{i+1} \cdot \frac{h_{s r_{i}}^{*}}{\left|h_{s r_{i}}\right|} \cdot r_{r_{i} \iota} \\
& r_{d}=\frac{h_{s d}^{*}}{\left|h_{s d}\right|} \cdot r_{s d}+\sum_{i=1}^{N} \frac{h_{s r_{i}}^{*}}{\left|h_{s r_{i}}\right|} \cdot r_{r_{i} d}
\end{aligned}
$$

Já a SNR instantânea da combinação pode ser definida como (COSTA; AISSA, 2009):

$$
\gamma_{E G C}=\frac{1}{N+1} \cdot\left[\sqrt{\gamma_{s d}}+\sum_{i=1}^{N} \sqrt{\gamma_{r_{i} d}}\right]
$$

considerando que o ruído de fundo possua a mesma variância nos caminhos fonte-destino e fonte-relaydestino.

O método EGC possui resultados superiores aos atingidos pelo SC (PROAKIS, 2001), porém às custas de aumento de complexidade devido ao processamento e detecção de todos os sinais transmitidos.

\subsection{Combinação de Máxima Razão}

Em termos de SNR, o método de combinação de máxima razão (MRC - Maximal-ratio Combining) é o mais eficiente para o aproveitamento de diversidade. A metodologia de combinação dos sinais no destinatário é basicamente a mesma do método EGC, porém os pesos não são igualitários, mas sim mensurados de acordo com a SNR instantânea alcançada, como pode ser visto na equação a seguir:

$$
\begin{gathered}
w_{1}=\left|h_{s d}\right| \\
w_{i+1}=\left|h_{r_{i} d}\right|, i=1,2, \cdots, N
\end{gathered}
$$

Definidos os pesos, o sinal a ser detectado será composto a partir da regra de combinação de máxima razão:

$r_{d}=h_{s d}^{*} \cdot r_{s d}+\sum_{i=1}^{N} h_{s r_{i}}^{*} \cdot r_{r_{i} d}$

O cálculo da SNR instantânea para o método MRC é dado por (COSTA; AISSA, 2009):

$\gamma_{M R C}=\gamma_{s d}+\sum_{i=1}^{N} \gamma_{r_{i} d}$

O método MRC é o método mais eficiente de combinação de diversidade (PROAKIS 2001), porém tem como principal dificuldade a alta 
complexidade não só devida ao processamento e detecção de todos os sinais, mas também no cálculo dos pesos, que devem ser atualizados a uma taxa igual ou maior ao inverso do tempo de coerência do canal.

\section{Resultados Numéricos de Simulação}

Para a simulação computacional e análise numérica das técnicas propostas foi utilizado o simulador matemático MATLAB da Mathworks, pois este constitui uma plataforma de simulação computacional completo com inúmeras facilidades na realização de cálculos vetoriais e matriciais, além de uma vasta biblioteca matemática de grande utilidade na análise numérica das mais variadas figuras de mérito de sistemas de comunicação sem fio.

Os dois protocolos discutidos foram analisados via simulação computacional, utilizando tanto a modelagem básica quanto aquela com uso de SNR de limiares (thresholds), os quais são empregados apenas na etapa de decisão de retransmissão em cada relay. Foram empregados os métodos de combinação de sinal SC e MRC no destinatário, bem como avaliado a melhoria de desempenho quando se utiliza três relays em relação à topologia de rede cooperativa com apenas um relay.

\section{Topologia de rede adotada}

A topologia adotada para rede cooperativa consiste de $\mathrm{N}$ relays, conforme disposto na figura 4. Considera-se que todos os caminhos apresentam desvanecimento independente do tipo Rayleigh plano, com média $\Omega_{s d}=\Omega_{s r_{i}}=\Omega_{r_{i} d}=1$, ruído de fundo AWGN com média igual a zero e variância dada por $\sigma_{r_{i}}^{2}=\sigma_{d}^{2}=\sigma^{2}$. A SNR média entre os nós é dada por $\Gamma_{s d}=\Gamma_{s r_{i}}=\Gamma_{r_{i} d}=\frac{\Gamma}{N+1}$ quando da utilização de métodos cooperativos e $\Gamma_{s d}=\Gamma \quad$ quando da utilização de métodos não cooperativos.
Figura 4. Topologia de rede proposta para simulação.

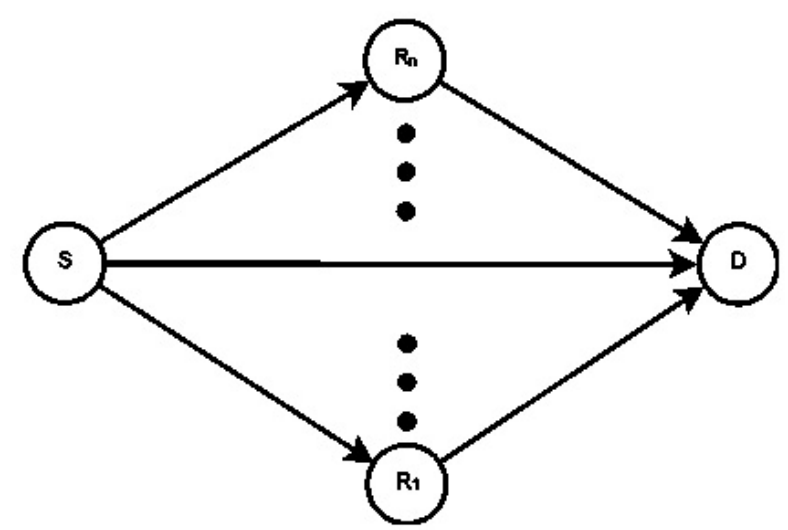

Visando verificar o impacto do número de relays no desempenho do sistema, cada protocolo será avaliado com a utilização de 1 e 3 relays. Para analisar o impacto da adoção de estratégias de minimização da propagação de erros, serão comparados os resultados obtidos com e sem a estratégia de utilização de um valor limiar para retransmissão, de acordo com as eqs. (8) e (11).

\section{Modelagem e Parâmetros de Simulação}

Utilizou-se modulação BPSK, acesso ao meio por divisão temporal (TDMA) com $N+1$ time-slots e detecção abrupta. Foram analisadas duas relevantes figuras de mérito: taxa de erro de bit e probabilidade de outage, ambas em relação à variação da SNR total por bit, $\Gamma$, a qual representa um dos parâmetros de entrada da simulação. Tais figuras de mérito foram escolhidas devido à complementaridade na análise que ambas possibilitam: a taxa de erro de bit é uma figura de mérito estreitamente relacionada à camada física, enquanto a probabilidade de outage é mais adequada para expressar desempenho da camada de controle de acesso ao meio (MAC). Para o cômputo da probabilidade de outage, define-se um valor de threshold, denotado por $\gamma^{*}$, o qual é comparado à SNR instantânea conforme demonstra a equação a seguir:

$$
\operatorname{outage}(\gamma)= \begin{cases}0, & \text { se } \gamma \geq \gamma^{*} \\ 1, & \text { se } \gamma<\gamma^{*}\end{cases}
$$


O processo de transmissão envolve a geração de bits aleatórios, que segue uma distribuição uniforme para os possíveis valores para o $i$-ésimo bit (0 e 1), sendo modulados utilizando a técnica BPSK. O mapeamento BPSK segue a seguinte regra:

$$
b_{i}=(-1)^{b i t_{i}}
$$

A descrição do coeficientes de canal de rádio móvel Rayleigh plano seguiu o modelo de Jakes (JAKES, 1974) modificado por Dent (DENT; BOTTOMLEY; CROFT, 1993). Neste modelo, a forma de onda contínua do desvanecimento é dada por:

$C(t)=\sqrt{\frac{2}{N_{\text {osc }}}} \cdot \sum_{n=1}^{N_{\text {osc }}}\left(e^{j \cdot \phi_{n}} \cdot \cos \left(w_{n} \cdot t+\theta_{n}\right)\right)$

com $N_{\text {osc }}=\frac{N_{d}}{4} \quad$ sendo o número de osciladores; $\phi_{n}=\frac{\pi \cdot n}{N_{o s c}} ; \theta_{\mathrm{n}}$ a fase inicial para cada um dos osciladores. Utilizando-se com distribuição uniforme no intervalo $[ \pm \pi]$ obtêm-se as formas de onda com distribuição Rayleigh para $|C(t)|$ e distribuição uniforme para os ângulos de $C(t)$.

A simulação do ruído AWGN é feita mantendose a amplitude do sinal normalizada e alterando a variância do processo de acordo com o valor da SNR média, conforme a seguinte equação:

$$
\sigma^{2}=\frac{A^{2}}{2 \cdot \frac{E_{b}}{N_{0}}}
$$

sendo $\frac{N_{0}}{2}$ a densidade espectral de potência bilateral do AWGN; $E_{b}=\mathrm{A}^{2} . T$ a energia de bit, $\operatorname{com} T$ o período de bit e $A$ a amplitude do sinal.

Tendo estes dados, a transmissão é simulada aplicando as eqs. (2) e (3). A técnica de transmissão TDMA é simulada no processo ao se fazer que cada dispositivo transmita o sinal em uma determinada sequência, no seu time-slot.
Como é pressuposto que os dispositivos possuem a capacidade de estimar perfeitamente os coeficientes de canal das transmissões nas quais estão envolvidos (i.e., não conhecem todos os coeficientes da rede), os resultados da eq. (21) podem ser utilizados no cálculo do fator de amplificação descrito na eq. (5) e também para a verificação do threshold de retransmissão $\gamma^{*}{ }_{\text {ri }}$, definido nas eqs. (8) e (11).

Para avaliar os resultados de maneira estatística, faz-se necessário que o experimento seja repetido por um determinado número de vezes, garantindo assim que os resultados não estejam diretamente associado ao determinado número ou condição de realização do experimento. Desta maneira, a simulação proposta utiliza o método de simulação Monte-Carlo para a definição do número de realizações do experimento probabilístico (trials). A probabilidade de erro de bit do sistema $\left(\mathrm{P}_{\mathrm{e}}\right)$, também denominada taxa de erro de bit pode ser calculada a partir do experimento probabilístico (MCS) como:

$$
B E R=P_{e}=\frac{n_{\text {erros }}}{\text { trials }}
$$

Utilizando a probabilidade de erro como a taxa de erro mínima para o caso de apenas 1 usuário (SuB - single-user bound), considerando modulação BPSK e canal Rayleigh plano, tem-se o desempenho analítico (PROAKIS, 2001):

$$
B E R=\frac{1}{2}\left(1-\sqrt{\frac{\Gamma}{1+\Gamma}}\right)
$$

Assim, pode-se prever o número de realizações necessário na simulação computacional MCS que produz um número de erros nerros médio, dado a SNR média de operação da rede de comunicação sob análise:

$$
\text { trials }=\frac{2 \cdot n_{\text {erros }}}{1-\sqrt{\frac{\Gamma}{1+\Gamma}}}
$$


Normalmente, o número de erros é definido como $\mathrm{n}_{\text {erros }} \geq 10$, de tal sorte a garantir um valor mínimo de confiança para o resultado de simulação MCS.

A tabela 1 resume os parâmetros utilizados na simulação MCS.

Tabela 1. Número de operações

\begin{tabular}{ll}
\hline Parâmetro & Valor \\
\hline Velocidade do móvel & $20 \mathrm{~km} / \mathrm{h}$ \\
Frequência da portadora & $f_{c}=3 \mathrm{GHz}$ \\
Número de erros & $\mathrm{n}_{\text {erros }}=100$ \\
Limiar de retransmissão & $5 \mathrm{~dB}$ \\
Limiar de outage & $\gamma_{\gamma^{*}}{ }_{\mathrm{i}}=5 \mathrm{~dB}$ \\
SNR média total & $\Gamma=[0-30] \mathrm{dB}$ \\
Número de relays & $\mathrm{N}=1$ ou 3 \\
\hline
\end{tabular}

\section{Bit Error Rate}

Os gráficos da Fig. 5 ilustram os resultados obtidos para a BER em relação à variação da SNR para o protocolo Amplifica e Transmite, sendo o primeiro com a utilização de 1 relay e o segundo com 3 relays. Os resultados demonstram que a técnica de combinação de sinal MRC é eficiente no aproveitamento da diversidade propiciada pelo protocolo Amplifica e Transmite, porém a amplificação de ruído propiciada por este método acaba por inviabilizar o método de combinação por seleção.

Para o protocolo Decodifica e Transmite, os gráficos da Fig.6 demonstram que técnicas de minimização da probabilidade de propagação de erro por parte dos relays são necessárias, pois os resultados obtidos no máximo igualam o desempenho da utilização de apenas o caminho direto (além da perda devido ao compartilhamento da SNR total por todos os caminhos, resultados experimentais demonstram que o sinal retransmitido sofre com uma redução equivalente a cerca de $5 \mathrm{~dB}$ devido aos erros de detecção).

Os gráficos da Fig. 7 demonstram os resultados para o protocolo Amplifica e Transmite com a adoção do SNR de threshold para retransmissão $\gamma \gamma^{*}{ }_{i}$, conforme definido na eq. (8). Os resultados demonstram a eficácia da adoção desta estratégia, obtendo resultados muito superiores em relação aos obtidos sem a utilização de nenhuma técnica de redução de propagação de erros. Ainda é possível concluir que, pelo menos quando da utilização do $\mathrm{MRC}$, o aumento do número de relays teve significante impacto na redução da BER.

A mesma estratégia foi adotada também para o protocolo Decodifica e Transmite, sendo os resultados ilustrados nos gráficos da Fig. 8. Assim como ocorreu para o caso anterior, a adoção do threshold possibilitou resultados mais promissores, reduzindo de maneira efetiva a BER tanto em relação à abordagem não cooperativa quanto ao protocolo sem a adoção de threshold.

\section{Probabilidade de outage}

Os gráficos da Fig. 9 ilustram os resultados obtidos para o protocolo Amplifica e Transmite. Houve uma forte redução na probabilidade de outage, mesmo com a adoção de apenas 1 relay. Adicionalmente, observa-se um significativo aumento no ganho de diversidade propiciado pelo aumento no número de relays. Tal ganho de diversidade pode ser medido através da inclinação das curvas na região assintótica, i.e., na região de médio-alto SNR. 
Figura 5. BER em relação à variação da SNR para o protocolo Amplifica e Transmite, com utilização de 1 relay (esq) e 3 relays (dir).
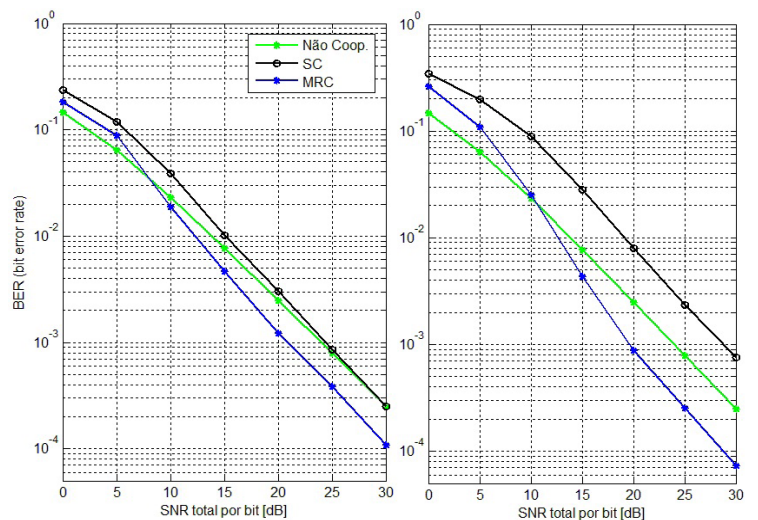

Figura 6. BER em relação à variação da SNR para o protocolo Decodifica e Transmite, com 1 relay (esq) e 3 relays (dir).
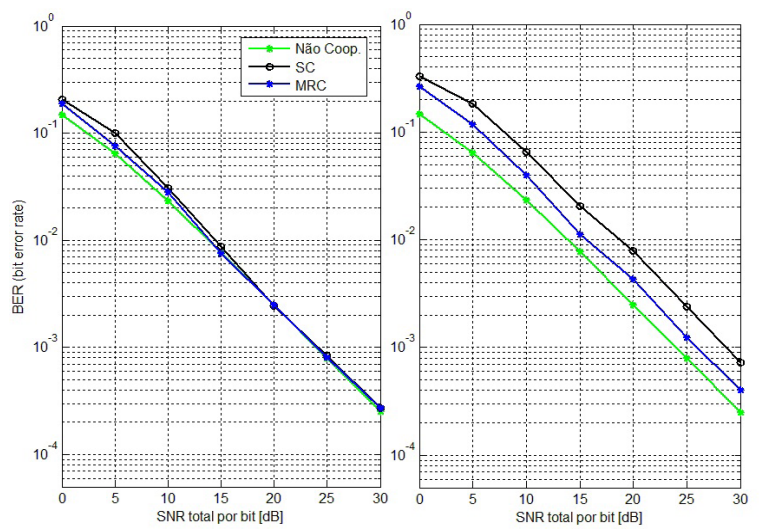

Figura 7. BER em relação à variação da SNR para o protocolo Amplifica e Transmite, com utilização de 1 relay (esq) e 3 relays (dir) e threshold de $5 \mathrm{~dB}$ para retransmissão.
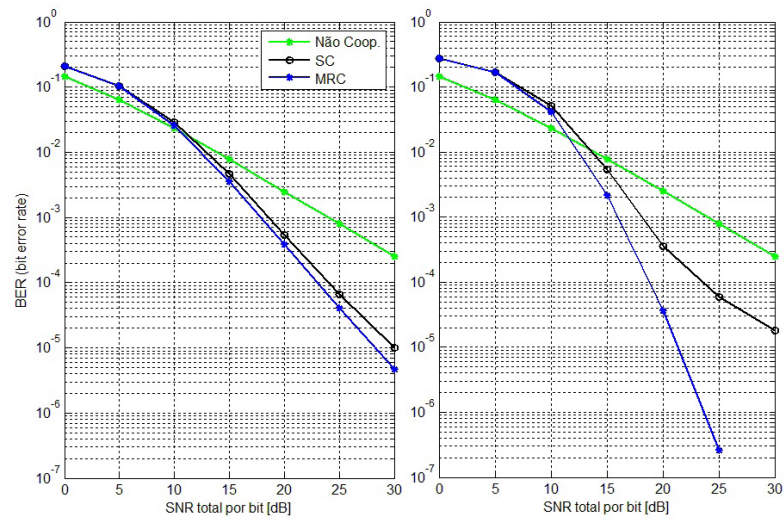

Figura 8. BER em relação à variação da SNR para o protocolo Decodifica e Transmite, com utilização de 1 relay (esq) e 3 relays (dir) e threshold de $5 \mathrm{~dB}$ para retransmissão.
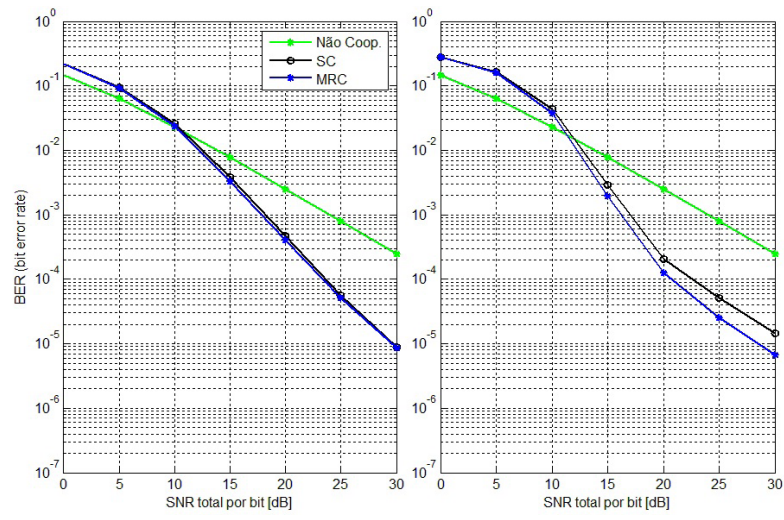

Para o protocolo Decodifica e Transmite, com resultados ilustrados na Fig.10, pode-se observar o potencial do protocolo e do próprio paradigma cooperativo na redução da probabilidade de outage, além da forte influência do número de relays no desempenho, mensurado pela inclinação da curva de desempenho na região de alto SNR.

Figura 9. Probabilidade de outage em relação à variação da SNR para o protocolo Amplifica e Transmite, com utilização de 1 relay (esq) e 3 relays (dir).
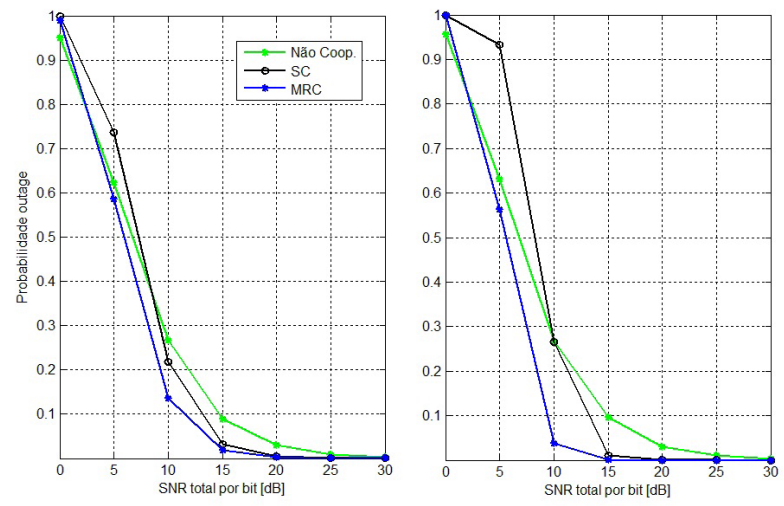

Tratando agora do protocolo Amplifica e Transmite com adoção de threshold (gráficos da Fig. 11), é possível perceber um aumento na probabilidade de outage para os dois métodos de combinação de sinal e independente do número de relay utilizados. Este aumento ocorre devido à não retransmissão de alguns dos sinais que não 
atingiram o threshold mínimo de retransmissão.

Assim como ocorrido para o caso anterior, a utilização do threshold de retransmissão para o protocolo Decodifica e Transmite incorreu em aumento na probabilidade de outage (Fig.12), especialmente visto quando da utilização do método MRC para valores mais baixos de SNR.

Figura 10. Probabilidade de outage em relação à variação da SNR para o protocolo Decodifica e Transmite, com utilização de 1 relay (esq) e 3 relays (dir).
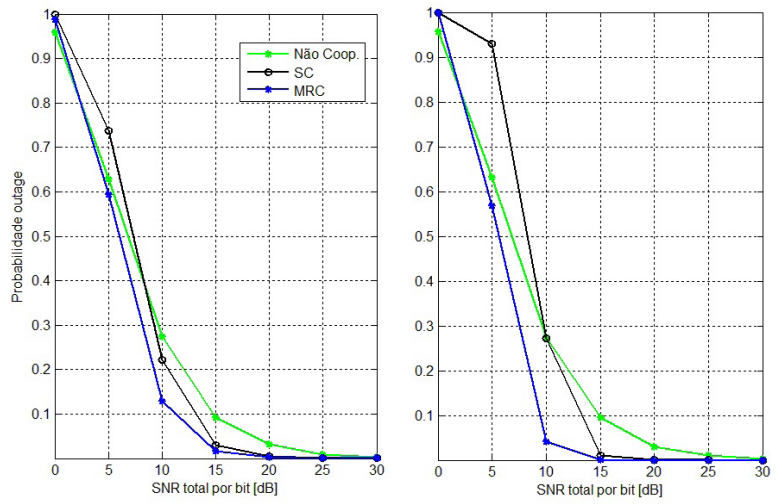

Figura 11. Probabilidade de outage em relação à variação da SNR para o protocolo Amplifica e Transmite, com utilização de 1 relay (esq) e 3 relays (dir) e threshold de $5 \mathrm{~dB}$ para retransmissão.
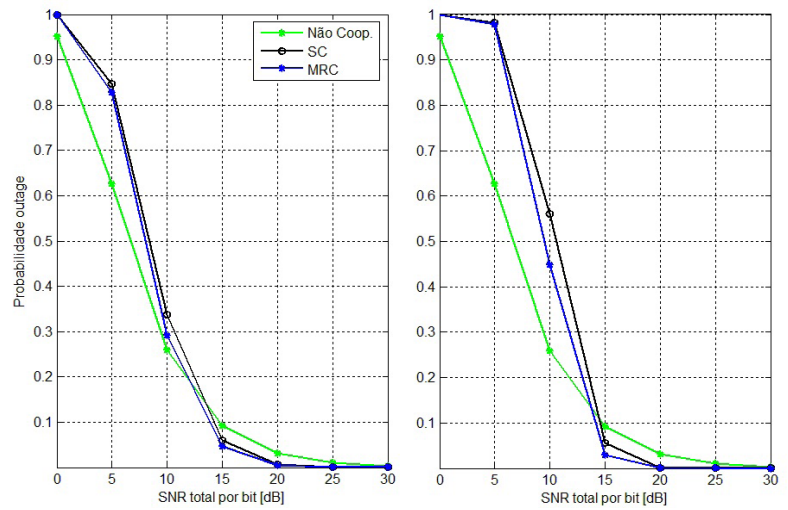

\section{Conclusões}

A abordagem cooperativa se mostrou de grande valia no combate às condições de propagação do canal rádio móvel, principalmente no que se refere à redução da probabilidade de outage. Em termos de redução da BER, resultados numéricos de simulação indicaram se faz necessidade de adoção de técnicas que reduzam a probabilidade de propagação de erro por parte dos relays. Neste sentido, a técnica adotada neste trabalho (estabelecimento de um threshold para retransmissão) mostrou-se efetiva na melhoria das figuras de mérito analisadas.

Finalmente, foi possível concluir que o método de combinação de sinais MRC é mais eficiente que o método SC na redução da probabilidade de outage e na BER para o protocolo Amplifica e Transmite com a utilização de um maior número de relays. No entanto, sob a condição de reduzido número de relays com a utilização do protocolo Decodifica e Transmite, a diferença de desempenho em termos de BER para os dois métodos não justifica a utilização do MRC, dada excessiva complexidade introduzida por esta regra de combinação.

Figura 12. Probabilidade de outage em relação à variação da SNR para o protocolo Decodifica e Transmite, com utilização de 1 relay (esq) e 3 relays (dir) e threshold de 5 dB para retransmissão.
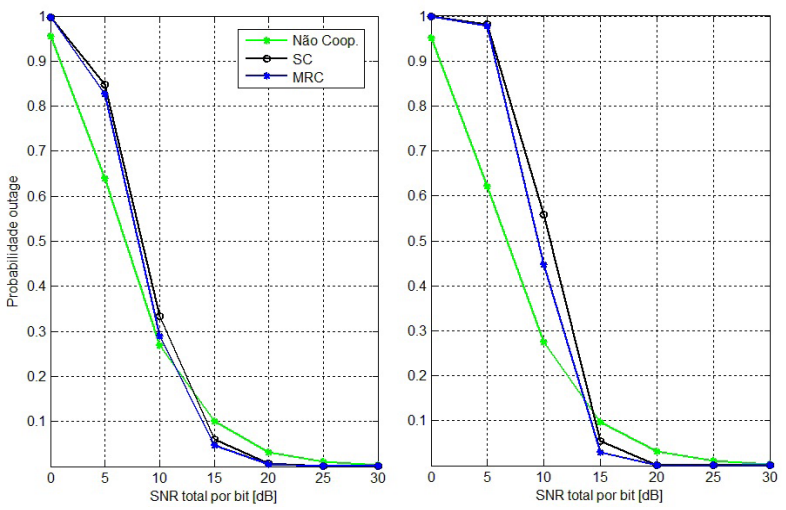

\section{Referências}

ALAZEM, F.; FRIGON, J.-F.; HACCOUN, D. Adaptive coded cooperation in wireless networks. In: INTERNATIONAL WIRELESS COMMUNICATIONS AND MOBILE COMPUTING CONFERENCE, 2008, Crete Island. Proceedings... Crete Island: [s.n.], 2008. p. 899-904.

COSTA, D. B.; AISSA, S. Performance of cooperative diversity networks: analysis of amplify-and-forward relaying under equalgain and maximal-ratio combining. In: INTERNATIONAL CONFERENCE ON 
COMMUNICATIONS, 2009, Dresden. Proceedings... Dresden: [s.n.], 2009. p. 1-5.

DENT, P.; BOTTOMLEY, G. E.; CROFT, T. Jakes fading model revisited. IEEE Electronics Letters, New York, n. 3, p. 1162-1163, 1993.

HASNA, M.; ALOUINI, M.-S. Performance analysis of two-hop relayed transmissions over rayleigh fading channels. In: VEHICULAR TECHNOLOGY CONFERENCE, 56., 2002, Vancouver. Proceedings... Vancouver: IEEE, 2002. p. 1992-1996.

HASNA, M.; ALOUINI, M.-S. A performance study of dual-hop transmissions with fixed gain relays. IEEE Transactions on Wireless Communications, New York, v. 3, n. 6, p. 1963-1968, 2004.

HUNTER, T.; NOSRATINIA, A. Diversity through coded cooperation. IEEE Transactions on Wireless Communications, New York, v. 5, n. 2, p. 283-289, 2006.

IKKI, S.; AHMED, M. Performance of cooperative diversity using equal gain combining (egc) over nakagami-m fading channels. IEEE Transactions on Wireless Communications, New York, v. 8, n. 2, p. 557$562,2009$.

JAHROMI, A.; AGHAEINIA, H.; DANESHMAND, S.; RAZAVIZADEH, S. On multi-user detection in cdma based cooperative networks. In: IEEE SARNOFF SYMPOSIUM, 2009, Princeton. Proceedings... Princeton: IEEE, 2009. p. 1-6.

JAKES, W. C. Microwave mobile communications. New York: Wiley, 1974.

KORAKIS, T.; KNOX, M.; ERKIP, E.; PANWAR, S. Cooperative network implementation using open-source platforms. IEEE Communications Magazine, Piscataway, v. 47, n. 2, p. 134-141, 2009.

KRAMER, G.; MARIC, I.; YATES, R. D. Cooperative communications. Foundations and Trends in Networking, Hanover, v. 1, n. 3, p. 271-425, 2006.

LANEMAN, J.; WORNELL, G. Energy-efficient antenna sharing and relaying for wireless networks. In: IEEE WIRELESS COMMUNICATIONS AND NETWORKING CONFERENCE, 2000, Chicago. Proceedings... Chicago: IEEE, 2000. p. 7-12.

LANEMAN, J.; WORNELL, G.; TSE, D. An efficient protocol for realizing cooperative diversity in wireless networks. In: IEEE INTERNATIONAL SYMPOSIUM ON INFORMATION THEORY, 2001, Washington. Proceedings... Washington: IEEE, 2001. p. 294.

LEE, I.-H.; KIM, D. Ber analysis for decode-andforward relaying in dissimilar rayleigh fading channels. IEEE Communications Letters, New York, v. 11, n. 1, p.
52-54, 2007.

NOSRATINIA, A.; HUNTER, T.; HEDAYAT, A. Cooperative communication in wireless networks. IEEE Communications Magazine, Piscataway, v. 42, n. 10, p. 74-80, 2004.

PROAKIS, J. G. Digital Communications. New York: McGraw-Hill, 2001.

QUOC, B. V. N.; KONG, H. Y.; DINH, C. H.; TIEN, T. L. Ber performance of decode-and-forward relaying using selection combining over rayleigh fading channels. In: INTERNATIONAL CONFERENCE ON ADVANCED TECHNOLOGIES FOR COMMUNICATIONS, 2008, Hanoi. Proceedings... Hanoi: IEEE, 2008. p. 301-304.

RODOPLU, V.; MENG, T. H. Minimum energy mobile wireless networks. IEEE Journal on Selected Areas in Communications, Piscataway, v. 17, n. 8, p. 1333-1344, 1999.

SELVARAJ, M. D.; MALLIK, R. K. Performance analysis of a multi-relay cooperative diversity network with decode and forward relaying. In: NATIONAL CONFERENCE ON COMMUNICATIONS, 2010, Chennai. Proceedings... Chennai: IEEE, 2010. p. 1-5.

SIRIWONGPAIRAT, W.; SADEK, A.; LIU, K. Cooperative communications protocol for multiuser ofdm networks. IEEE Transactions on Wireless Communications, New York, v. 7, n. 7, p. 2430-2435, 2008.

STALLINGS, W. Wireless communications \& networks. New York: Prentice Hall, 2005.

TORABI, M.; HACCOUN, D.; AJIB, W. Performance analysis of cooperative diversity with relay selection over non-identically distributed links. Communications, IET, New York, v. 4, n. 5, p. 596-605, 2010.

VIEN, N.; NGUYEN, H. Performance analysis of fixedgain amplify-and-forward relaying with mrc. IEEE Transactions on Vehicular Echnology, New York, v. 59, n. 3, p. 1544-1552, 2010.

XU, W.; ZHANG, J.; ZHANG, P. Outage probability of dual-hop fixed-gain relay with unequal-power rayleigh interference. IEEE Communication Letters, New York, v. 15 , n. 6, p. 608-610, 2010.

YAN, Q.; YUE, D.-W.; HE, Y. Cooperative diversity of wireless networks with multiple amplify-andforward relays and hard-decision detections. In: INTERNATIONAL CONFERENCE ON COMMUNICATIONS AND MOBILE COMPUTING, 2010, Shenzhen. Proceedings... Shenzhen: IEEE, 2010. p. 207-212. v. 2.

ZHANG, Y.; MA, Y.; TAFAZOLLI, R. Improved coded 
cooperation schemes for wireless communications. In: INTERNATIONAL SYMPOSIUM ON MODELING AND OPTIMIZATION IN MOBILE, AD HOC, AND WIRELESS NETWORKS AND WORKSHOPS, 6., 2008, Berlin. Proceedings... Berlin: IEEE, 2008. p. 533538.

ZHANG, Y.; SHENG, M.; LI, J.; TIAN, Y.; YAO, J.; TANG, D. Optimal resource allocation for energy efficient transmissions with qos constrains in coded cooperative networks. In: IEEE CONFERENCE ON WIRELESS COMMUNICATIONS \& NETWORKING CONFERENCE, 2009, Piscataway. Proceedings... Piscataway: IEEE, 2009. p. 1658-1663.

ZHOU, Z.; ZHOU, S.; CUI, J.; CUI, S. Energy-efficient cooperative communication based on power control and selective single-relay in wireless sensor networks. IEEE Transactions on Wireless Communications, New York, v. 7, n. 8, p. 3066-3078, 2008.

Recebido em 3 Março 2011-Received on March 3, 2011.

Aceito em 26 Abril, 2011 - Accepted on April 26, 2011. 
